\title{
A NOTE ON THE CONSTRUCTION OF A CERTAIN CLASS OF KLEINIAN GROUPS
}

\author{
RICARDO BIANCONI
}

(Communicated by Peter $\mathrm{Li}$ )

\begin{abstract}
We prove that if $\left\{S_{1}, S_{1}^{\prime}, \ldots, S_{n}, S_{n}^{\prime}\right\}$ is a collection of distinct spheres in $\mathbb{R}^{m}$ with common exterior, and $g_{1}, \ldots, g_{n}$ are Möbius transformations such that for each $i, S_{i}$ is the isometric sphere of $g_{i}$ and $S_{i}^{\prime}$ is the isometric sphere of $g_{i}^{-1}$ and such that $g_{i}$ maps points of contact of $S_{i}$ to points of contact of $S_{i}^{\prime}$, then the group $G$ generated by the $g_{i}$ 's is Kleinian.
\end{abstract}

\section{INTRODUCTION}

A practical way to construct examples of Kleinian groups (acting in the real hyperbolic space as a group of isometries or in $\overline{\mathbb{R}}^{m}=\mathbb{R}^{m} \cup\{\infty\}$ as a group of conformal transformations) is to choose spheres $\left\{S_{1}, S_{1}^{\prime}, \ldots, S_{n}, S_{n}^{\prime}\right\}$ in $\overline{\mathbb{R}}^{m}$ and Möbius transformations $g_{1}, \ldots, g_{n}$ such that $g_{i}$ maps the outside of $S_{i}$ to the inside of $S_{i}^{\prime}$. But this is not enough to guarantee that the group generated by $g_{1}, \ldots, g_{n}$ is Kleinian.

In [1] M. Bestvina and D. Cooper intended to give an example of a Kleinian group whose limit set in $S^{3}$ is a wild Cantor set using this idea. But, as was pointed out in [2], there is a gap in their result because it is not verified there whether the group obtained is Kleinian. In section 2.5 we give an example due to B. Maskit which shows that this technique must be used carefully.

In this paper we show that if we take care of some symmetry conditions and a particular choice of the Möbius transformations the group obtained is Kleinian. Basically we show that in this case some parabolicity condition is satisfied, thus making unnecessary the (sometimes troublesome) algebraic verification.

In section 2 we present the basic definitions and Poincaré's Polyhedron Theorem (in a version sufficient to our purposes) and then define the particular setting for the construction of a Kleinian group. Then in section 3 we state precisely what needs to be proved and then sketch the proof of this fact. In

Received by the editors March 28, 1994.

1991 Mathematics Subject Classification. Primary 30F40, 20H10.

Key words and phrases. Kleinian groups, parabolic, infinite cycle transformations, isometric spheres, Poincaré's Polyhedron Theorem.

The author was partially supported by $\mathrm{CNP}_{\mathrm{q}}$. 
section 4 we prove the main result. And in section 5 we discuss some future work that can be done in these lines.

\section{Preliminaries}

2.1. Let us first set the terminology. We will follow Maskit's book [3].

We can look at a Möbius transformation either as an isometry of the hyperbolic space $\mathbb{H}^{m+1}$ or as a conformal transformation of its boundary at infinity, which can be modelled by $\overline{\mathbb{R}}^{m}=\mathbb{R}^{m} \cup\{\infty\}$.

If a Möbius transformation $g$ does not fix $\infty$, then there is a Euclidean sphere $S$ centered at $g^{-1}(\infty)$ which is mapped by $g$ to a sphere $S^{\prime}$ of the same radius centered at $g(\infty)$. The sphere $S$ is called the isometric sphere of $g$. It can be proved that $g$ can be decomposed as $g=q \circ p$ where $p$ is the inversion in $S$ and $q$ is a Euclidean motion (see [3, Chapter 4, section G]).

A Kleinian group is a group of Möbius transformations which acts properly discontinuously at some open set in $\mathbb{H}^{m+1}$ or in $\overline{\mathbb{R}}^{m}$.

A (finite) polyhedron $F^{\prime}$ in $\mathbb{H}^{m+1}$ is a nonempty open region bounded by finitely many hyperbolic hyperplanes (which are called the sides of $F^{\prime}$ ). Their boundaries or traces in $\overline{\mathbb{R}}^{m}$ are either Euclidean spheres or hyperplanes (i.e., spheres passing through $\infty)$. So these define a region $F$ in $\overline{\mathbb{R}}^{m}$ which is the trace of $F^{\prime}$. An edge is the intersection of two sides of $F^{\prime}$. An edge at infinity is the tangency point of the trace of two sides which do not intersect but have distance zero. We define the angle $\alpha(e)$ of an edge $e$ as the angle between the sides defining it measured from the inside of $F^{\prime}$.

2.2. Assume that for each side $s$ of $F^{\prime}$ there is a given Möbius transformation $g_{s}$ such that there is a side $s^{\prime}$ of $F^{\prime}$ such that $g_{s}(s)=s^{\prime}$. These $g_{s}$ are called side pairing transformations of $F^{\prime}$.

Observe that edges are mapped to edges by the side pairing transformations so they come in cycles under their action. The same happens to the edges at infinity. Let $\left\{e_{1}, \ldots, e_{k}\right\}$ be a cycle of edges (respectively edges at infinity) and let $\left\{g_{1}, \ldots, g_{k}\right\}$ be side pairing transformations such that $g_{i}\left(e_{i}\right)=e_{i+1}$ if $1 \leq i<k$ and $g_{k}\left(e_{k}\right)=e_{1}$. Let $h_{e}=g_{k} \circ \cdots \circ g_{1}$. This is called cycle transformation of the edge $e=e_{1}$ (respectively infinite cycle transformation of the edge at infinity $e$ ).

Let $G$ be the group generated by the $g_{s}$ (for all sides $s$ of $F^{\prime}$ ).

\subsection{Consider the following conditions:}

1. $g_{s}(s)=s^{\prime}$;

2. $g_{s^{\prime}}=g_{s}^{-1}$;

3. $g_{s}\left(F^{\prime}\right) \cap F^{\prime}=\varnothing$;

4. the orbit under $G$ of any $x \in \bar{F}^{\prime}$ has only finitely many points in $\bar{F}^{\prime}$;

5. for each edge $e$ there is a positive integer $t$ such that $h_{e}^{t}=1$;

6. for each cycle of edges $\left\{e_{1}, \ldots, e_{k}\right\}$ let $t$ be the minimal $i$ such that $h_{e}^{i}=1, e=e_{1} ;$ then $\sum_{j=1}^{k} \alpha\left(e_{j}\right)=2 \pi / t$;

7. the infinite cycle transformation at an infinite edge is parabolic.

Then

Theorem 1 (Poincaré's Polyhedron Theorem). If the conditions (1) through (7) above are satisfied, then the group $G$ is Kleinian and $F^{\prime}$ is a fundamental domain for $G$. 
By [3, Chapter 6, section A.3], the trace $F$ of $F^{\prime}$ in $\overline{\mathbb{R}}^{m}$ is a fundamental domain for $G$ acting on $\overline{\mathbb{R}}^{m}$ as a group of conformal transformations. Of course we can extend the action of a group of conformal transformations of $\overline{\mathbb{R}}^{m}$ to $\mathbb{H}^{m+1}$. If this extended action gives a Kleinian group, then the original one also gives a Kleinian group.

So, consider the following setting.

2.4. Let $\mathscr{B}=\left\{B_{1}, B_{1}^{\prime}, \ldots, B_{n}, B_{n}^{\prime}\right\}$ be a collection of closed metric balls in $\mathbb{R}^{m}$, such that:

1. the radius of $B_{i}$ equals the radius of $B_{i}^{\prime}$, for all $i$;

2. each ball lies outside the others, that is, if $i \neq j, \operatorname{int}\left(B_{i}\right) \cap \operatorname{int}\left(B_{j}\right)=$ $\operatorname{int}\left(B_{i}^{\prime}\right) \cap \operatorname{int}\left(B_{j}^{\prime}\right)=\varnothing$, and for all $k, l, \operatorname{int}\left(B_{k}\right) \cap \operatorname{int}\left(B_{l}^{\prime}\right)=\varnothing$;

3. they can touch.

Put $S_{i}=\partial B_{i}$ and $S_{i}^{\prime}=\partial B_{i}^{\prime}$.

Let $g_{1}, \ldots, g_{n}$ be (orientation-preserving) Möbius transformations such that:

- for each $i, S_{i}$ is the isometric sphere of $g_{i}$ and $S_{i}^{\prime}$ is the isometric sphere of $g_{i}^{-1}$

- $g_{i}$ maps points of contact of $S_{i}$ to points of contact of $S_{i}^{\prime}$.

We are going to prove that the group $G$ generated by the $g_{i}$ 's is a Kleinian group. We observe that the conditions (1) to (6) are automatically satisfied. Conditions (5) and (6) are satisfied because there are no edges other than the infinite ones in this setting. We need only to verify if the infinite cycle transformations at every infinite edge are parabolic.

2.5. An example. We call attention to the following example due to Maskit [4], which shows that this verification is really necessary.

Let $S_{1}=\{z \in \mathbb{C}:|z|=1\}, S_{1}^{\prime}=\{z \in \mathbb{C}:|z|=3\}, S_{2}=\{z \in \mathbb{C}:|z+2|=1\}$ and $S_{2}^{\prime}=\{z \in \mathbb{C}:|z-2|=1\}$, and $g_{1}(z)=3 z, g_{2}(z)=(2 z+5) /(z+2)$. It is easy to verify that $S_{2}$ is the isometric circle of $g_{2}$ and $S_{2}^{\prime}$ the isometric circle of $g_{2}^{-1}$. But we cannot speak of isometric circles of $g_{1}$ and $g_{1}^{-1}$ because they fix $\infty$. Let $D$ be the region bounded by these spheres, i.e. between $S_{1}$ and $S_{1}^{\prime}$ and outside $S_{2}$ and $S_{2}^{\prime}$. This setting satisfies conditions (1) through (6) but not the parabolicity condition (7). (The infinite cycle transformation of the contact point $P$ of $S_{1}$ and $S_{2}$ is $h=g_{1}^{-1} g_{2}^{-1} g_{1}^{-1} g_{2}$; the reader can verify that $h$ is loxodromic, not parabolic.) In fact, Maskit shows that $D$ is not a fundamental domain for the group generated by $g_{1}$ and $g_{2}$.

So we stress the importance of the hypothesis that the spheres $S_{i}$ and $S_{i}^{\prime}$ be the isometric spheres of $g_{i}$ and $g_{i}^{\prime}$ respectively, in our result.

\section{Statement of the Result AND SKetch OF THE PROOF}

3.1. Let $h=a_{k} a_{k-1} \ldots a_{1}$ be an infinite cycle transformation at an infinite edge (or contact point), where $a_{i} \in\left\{g_{1}, \ldots, g_{n}\right\} \cup\left\{g_{1}^{-1}, \ldots, g_{n}^{-1}\right\}$. Let $I_{j}$ and $I_{j}^{\prime}$ be the isometric spheres of $a_{j}$ and $a_{j}^{-1}$ respectively. We have that each $a_{j}$ can be decomposed as $a_{j}=q_{j} p_{j}$ where $p_{j}$ is the inversion in $I_{j}$ and $q_{j}$ is an euclidean motion. (See [3, Chapter 4 , section G].) For each $j=1, \ldots, k$, let $R_{j}, R_{j}^{\prime}, T_{j}, T_{j}^{\prime}$ denote the open regions inside $I_{j}$ and $I_{j}^{\prime}$ and outside $I_{j}$ and $I_{j}^{\prime}$ respectively. 
Thus $h$ is a cycle transformation of the contact point $X_{1}$ of $I_{1}$ and $I_{k}^{\prime}$. We want to show that

Theorem 2. $X_{1}$ is the only fixed point of $h$ in $\mathbb{R}^{n} \cup\{\infty\}$ (and hence $h$ is parabolic).

3.2. We first show that there is no fixed point of $h$ in $T_{1} \cup T_{k}^{\prime} \cup\{\infty\}$ (the region outside the isometric spheres $I_{1}$ and $I_{k}^{\prime}$ ) by showing that the image under $h$ of $T_{1} \cup T_{k}^{\prime} \cup\{\infty\}$ lies inside $R_{k}^{\prime}$, which is disjoint of this region. Next we show that there is no fixed point of $h$ in $R_{k}^{\prime}$ (the region inside $I_{k}^{\prime}$ ). This we do by showing that the image under $h$ of any sphere $S \subseteq \bar{T}_{1}$ (i.e., not inside $I_{1}$ ), tangent to $I_{1}$ at $X_{1}$, has diameter strictly less than the diameter of $S$. The same argument then applies to show that there is no fixed point of $h^{-1}$ in $R_{1}$ (the region inside $I_{1}$ ). Since the fixed points of $h^{-1}$ are exactly those of $h$, this proves that the only fixed point of $h$ is $X_{1}$. Hence $h$ is parabolic.

\section{THE PROOF}

We divide the proof in the following lemmas proving the three steps sketched above. Recall the decomposition $h=a_{k} a_{k-1} \cdots a_{1}$, with $a_{i} \in\left\{g_{1}, \ldots, g_{n}\right\} \cup$ $\left\{g_{1}^{-1}, \ldots, g_{n}^{-1}\right\}$. Warning: All the metric notions used here (e.g., sphere, its center, its diameter, etc.) are Euclidean.

4.1. Lemma. There is no fixed point of $h$ in $T_{1} \cup T_{k}^{\prime} \cup\{\infty\}$ (the region outside the isometric spheres $I_{1}$ and $\left.I_{k}^{\prime}\right)$.

Proof. Let $U_{0}=T_{1} \cup T_{k}^{\prime} \cup\{\infty\}$. Then inversion in $I_{1}$ maps $U_{0}$ into $R_{1}$. So $a_{1}$ maps $U_{0}$ into $R_{1}^{\prime}$ (because of the decomposition of $a_{1}=q_{1} p_{1}$, with $p_{1}$ the inversion in $I_{1}$ and $q_{1}$ a Euclidean motion which maps $R_{1}$ into $\left.R_{1}^{\prime}\right)$. But $R_{1}^{\prime}$ lies outside $I_{2}$, hence $a_{2}$ maps $U_{1}=a_{1}\left(U_{0}\right)$ into $R_{2}^{\prime}$. Inductively we have that if $U_{i}=a_{i}\left(U_{i-1}\right)$, then $U_{i} \subseteq R_{i}^{\prime}$. But $U_{k}=h\left(U_{0}\right)$. This proves the lemma.

4.2. Lemma. If $A_{1}$ is a sphere of diameter $d_{1}>0$ tangent to $I_{1}$ at $X_{1}$ and lying not inside $I_{1}$, then the diameter of $h\left(A_{1}\right)$ is strictly less than $d$. (Observe that $h\left(A_{1}\right)$ is a sphere lying not inside $I_{1}$ and tangent to $I_{1}$ at $X_{1}$; see Figure 1.)

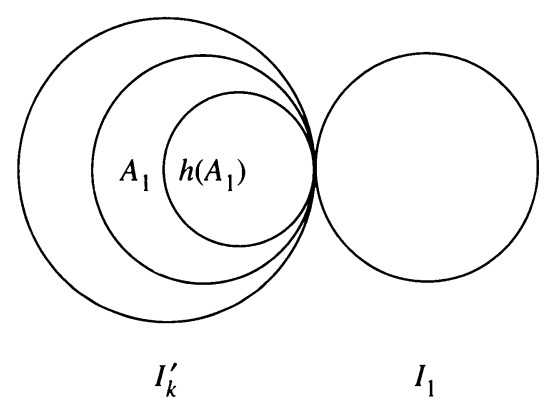

Figure 1. Statement of Lemma 4.2

Proof. We do this inductively by showing that the image under $a_{i}$ of a sphere $A_{i}$ of diameter $d_{i}>0$ tangent to $I_{1}$ at $X_{i}=a_{i-1}\left(X_{i-1}\right)$ (this is the point of contact of $I_{i-1}^{\prime}$ and $\left.I_{i}\right)$ is a sphere of diameter $d_{i+1}<d_{i}$ tangent to $I_{i+1}$ 
at $X_{i+1}=a_{i}\left(X_{i}\right)$ (and making $I_{k+1}=I_{1}$, this proves that the diameter $d_{k}$ of $h(B)$ is $\left.d_{k}<d_{1}\right)$.

So let $r_{i}$ be the radius of $I_{i}$. Then the diameter of $p_{i}\left(A_{i}\right)$ (the inversion of $A_{i}$ in $\left.I_{i}\right)$ is $d_{i+1}=r_{i}-r_{i}^{2} /\left(r_{i}+d_{i}\right)$. We have that:

$$
d_{i}-d_{i+1}=d_{i}-\left(r_{i}-\frac{r_{i}^{2}}{r_{i}+d_{i}}\right)=\frac{d_{i}^{2}}{r_{i}+d_{i}}>0,
$$

i.e., $d_{i+1}<d_{i}$. (See Figure 2.)

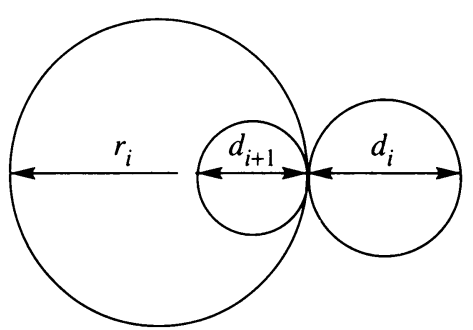

FIGURE 2. Comparison of diameters in the proof of Lemma 4.2

But $a_{i}\left(A_{i}\right)=q_{i} p_{i}\left(A_{i}\right)$ and $q_{i}$ is a Euclidean motion mapping $R_{i}$ into $R_{i}^{\prime}$. This finishes the proof of the lemma.

4.3. Lemma. There is no fixed point of $h$ in $I_{k}^{\prime} \cup R_{k}^{\prime}$ apart from $X_{1}$. (Observe that $X_{1} \in I_{k}^{\prime} \cap I_{1}$.)

Proof. Let $X \in I_{k}^{\prime} \cup R_{k}^{\prime}, X \neq X_{1}$. Let $A_{1}$ be the sphere containing $X$ and tangent to $I_{1}$ at $X_{1}$, and $d_{1}$ its radius. We observe that this sphere is unique. By Lemma 4.2 above, the radius of $h\left(A_{1}\right)$ is strictly less than $d_{1}$. Hence $X$ cannot be in $h\left(A_{1}\right)$. (Actually, the only common point of $A_{1}$ and $h\left(A_{1}\right)$ is $X_{1}$, the point of tangency.) Therefore $h(X) \neq X$.

4.4. Lemma. There is no fixed point of $h$ in $I_{1} \cup R_{1}$ apart from $X_{1}$.

Proof. The same proof of the previous lemma applies to prove that there is no fixed point of $h^{-1}$ in $I_{1} \cup R_{1}$. Since the fixed points of $h^{-1}$ are exactly the same as the ones of $h$, this proves the lemma.

With this we have finished the proof of Theorem 2.

\section{CONCLUSION}

By our result if we want to construct an example of a Kleinian group by taking touching spheres we need only to take care of using isometric spheres and to be sure that contact points go to contact points.

5.1. We believe that this construction can be useful to study deformations of this class of groups by simply describing their fundamental regions in terms of isometric spheres.

We ask the following questions.

Question 1. How does a deformation of $G$ correspond to a modification of $F$ ? 
Question 2. Can one reduce the problem of finding the dimension of the space of deformations of $G$ to a problem of linear algebra? This means, can one find an algebraic or combinatorial invariant related to this space of deformations?

\section{ACKNOWLEDGMENTS}

The author wishes to thank Dr. Elisha Falbel for the critical check of the correctness of the main result and Dr. Nikolay Gusevskii for introducing the author to this fascinating subject and for pointing out a serious mistake in a previous version of this result.

\section{REFERENCES}

1. M. Bestvina and D. Cooper, A wild Cantor set as the limit set of a conformal group action on $S^{3}$, Proc. Amer. Math. Soc. 99 (1987), 623-626.

2. R. Bianconi, N. Gusevskii, and H. Klimenko, Schottky-type groups and Kleinian groups acting on $\mathbb{R}^{3}$, preprint, 1994.

3. B. Maskit, Kleinian groups, Springer-Verlag, Berlin, 1988.

4. _ On Klein's combination theorem, Trans. Amer. Math. Soc. 120 (1965), 499-509.

IME-USP, Caixa Postal 66.281, CEP 05389-970, São Paulo, SP, Brazil

E-mail address: bianconi@ime.usp.br 